\title{
Prognostic power of anaerobic threshold parameters in patients with transposition of the great arteries and systemic right ventricle
}

\section{Original Article}

Cite this article: Gonçalves AV, Mano $\mathrm{T}$, Agapito A, Rosa SA, de Sousa L, Rio P, Alberto J, Monteiro A, da Silva TP, Moreira RI, Soares R, Pinto F, and Ferreira RC (2019) Prognostic power of anaerobic threshold parameters in patients with transposition of the great arteries and systemic right ventricle. Cardiology in the Young, page 1 of 7. doi: 10.1017/

S1047951119002361

Received: 6 May 2019

Revised: 25 August 2019

Accepted: 31 August 2019

\section{Keywords:}

Transposition of the great arteries; systemic right ventricle; heart failure; prognostic; Senning/Mustard

\section{Author for correspondence:}

A. V. Gonçalves, Department of Cardiology, Hospital de Santa Marta, Rua de Santa Marta, no 50, 1169-024 Lisbon, Portugal. Tel: +351 961156697; E-mail: antonio.a.goncalves.14@ gmail.com

\section{António V. Gonçalves ${ }^{1}$ (), Tânia Mano ${ }^{1}$, Ana Agapito ${ }^{1}$, Sílvia A. Rosa ${ }^{1}$, Lídia de Sousa ${ }^{1}$, Pedro Rio ${ }^{1}$, José Alberto ${ }^{1}$, André Monteiro ${ }^{1}$, Tiago P. da Silva ${ }^{1}$, Rita I. Moreira ${ }^{1}\left(\mathbb{D}\right.$, Rui Soares ${ }^{1}$, Fátima Pinto ${ }^{2}$ and Rui C. Ferreira ${ }^{1}$}

${ }^{1}$ Department of Cardiology, Hospital de Santa Marta, Centro Universitário Hospitalar de Lisboa Central, Lisbon, Portugal and ${ }^{2}$ Reference Center for Congenital Heart Defects, Hospital de Santa Marta, Centro Universitário Hospitalar de Lisboa Central, Lisbon, Portugal

\begin{abstract}
Introduction: Both transposition of the great arteries (TGA) previously submitted to a Senning/ Mustard procedure and congenitally corrected TGA (cc-TGA) have the systemic circulation supported by the morphological right ventricle, thereby rendering these patients to heart failure events risk. The aim of this study was to evaluate cardiopulmonary exercise test parameters for stratifying the risk of heart failure events in TGA patients. Methods: Retrospective evaluation of adult TGA patients with systemic circulation supported by the morphological right ventricle submitted to cardiopulmonary exercise test in a tertiary centre. Patients were followed up for at least 1 year for the primary endpoint of cardiac death or heart failure hospitalisation. Several cardiopulmonary exercise test parameters were analysed as potential predictors of the combined endpoint and their predictive power were compared (area under the curve). Results: Cardiopulmonary exercise test was performed in 44 TGA patients (8 cc-TGA), with a mean age of $35.1 \pm 8.4$ years. The primary endpoint was reached by $10(22.7 \%)$ patients, with a mean follow-up of $36.7 \pm 26.8$ months. Heart rate at anaerobic threshold had the highest area under the curve value (0.864), followed by peak oxygen consumption $\left(\mathrm{pVO}_{2}\right)(0.838)$. Heart rate at anaerobic threshold $\leq 95 \mathrm{bpm}$ and $\mathrm{pVO}_{2} \leq 20 \mathrm{ml} / \mathrm{kg} / \mathrm{min}$ had a sensitivity of 87.5 and $80.0 \%$ and a specificity of 82.4 and $76.5 \%$, respectively, for the primary outcome. Conclusion: Heart rate at anaerobic threshold $\leq 95 \mathrm{bpm}$ had the highest predictive power of all cardiopulmonary exercise test parameters analysed for heart failure events in TGA patients with systemic circulation supported by the morphological right ventricle.
\end{abstract}

Transposition of the great arteries (TGA) is a CHD with an incidence of $0.02 \%$ per birth, ${ }^{1}$ while congenitally corrected TGA (cc-TGA) has a lower incidence of $0.003 \%$ per birth. ${ }^{2}$ Until the 1980 s, the Senning and Mustard procedures were commonly performed for atrial switch, leaving a systemic right ventricular prone to dysfunction. From the late 1980s and early 1990s onwards, TGA has been managed instead by arterial switch operations in which the ventriculoarterial discordance is rectified. ${ }^{3}$ Patients with uncorrected cc-TGA also have the systemic circulation supported by the morphological right ventricle, thereby rendering these patients to an increased risk for heart failure events. ${ }^{4}$

Several theories have been postulated for the progressive dysfunction of the systemic right ventricle, which include the solely longitudinal arrangement of the cardiomyocytes in the right ventricle versus the predominantly circumferential with the radial and longitudinal patterns of the left ventricle, ${ }^{5}$ an increase in myocardial fibrosis due to right ventricle pressure overload and hypertrophy ${ }^{6}$ and a decrease in coronary flow reserve..$^{7-9}$

Failure of the systemic right ventricle could be resolved by anatomic repair either in Senning and Mustard patients (conversion to switch operation) or in cc-TGA (double-switch operation). However, right ventricle dysfunction usually remains sub-clinical for decades and typically manifests during fourth and fifth decades of life, when surgery is no longer a successful option, leaving heart transplantation as the only long-term solution. ${ }^{10}$

The cardiopulmonary exercise test is a powerful predictor of mortality in heart failure patients and is used as the standard criterion for the need for heart transplantation, ${ }^{11}$ with peak oxygen consumption $\left(\mathrm{pVO}_{2}\right)$ and the relation between ventilation and $\mathrm{CO}_{2}$ production (VE/ $\mathrm{VCO}_{2}$ slope) as the most used risk assessment tools. ${ }^{12}$ Several cardiopulmonary exercise test studies had previously shown that adult CHD patients have reduced exercise capacity similar to non-congenital heart failure patients ${ }^{13-15}$ and that cardiopulmonary exercise test can be used to stratify the risk of heart failure events in adults with CHD similar to the heart failure patients. In the Diller study, ${ }^{13} \mathrm{CHD}$ patients with a $\mathrm{pVO}_{2}$ lower than $15.5 \mathrm{ml} / \mathrm{kg} / \mathrm{min}$ had a 2 -year event 
rate of $50 \%$ regarding death or heart failure hospitalisation, whereas those with a $\mathrm{pVO}_{2}$ superior to $27 \mathrm{ml} / \mathrm{kg} / \mathrm{min}$ had a 2 -year event rate of only $3 \%$.

The aim of this study was to evaluate several cardiopulmonary exercise test parameters for stratifying the risk of heart failure events in TGA or cc-TGA patients with systemic right ventricle. We hypothesised that cardiopulmonary exercise test parameters may provide optimal prognostic information on midterm heart failure events in this population.

To the best of our knowledge, this is the first study to address the use of cardiopulmonary exercise test to stratify the risk of heart failure events in solely TGA or cc-TGA patients with systemic right ventricle without mixing other CHD and is one of the first studies to compare several different cardiopulmonary exercise test parameters in only one type of adult CHD.

\section{Methods}

The investigation conforms to the principles outlined in the Declaration of Helsinki. The institutional ethics committee approved the study protocol and all patients provided consent for the use of the collected information.

\section{Patient population and study protocol}

The study included a single tertiary centre retrospective analysis of 44 consecutive adult TGA patients with systemic circulation supported by the morphological right ventricle (TGA patients with Senning or Mustard procedure and cc-TGA patients), referred for cardiopulmonary exercise test as part of their clinical evaluation between January 2010 and December 2017. Cardiopulmonary exercise test is requested in our TGA patients with systemic right ventricle at intervals from 3 to 5 years, even in asymptomatic patients to detect early changes in exercise capacity. If one patient performed more than one cardiopulmonary exercise test during the time of evaluation, the last one was used for the analysis. Clinical, laboratorial, and echocardiographic information were also collected at the time of cardiopulmonary exercise test when available.

Patients were considered cyanotic if they had an oxygen saturation lower than $90 \%$ at rest.

\section{Follow-up and endpoint}

In order to stratify the risk of heart failure events in TGA patients using cardiopulmonary exercise test parameters, all patients were followed up for at least 12 months from the date of completion of the cardiopulmonary exercise test. The primary endpoint was a composite of cardiac death or heart failure hospitalisation. Data were obtained from the outpatient clinic visits and medical charts review and were complemented with a standardised telephone interview to all patients at the end of follow-up. Information regarding all-cause mortality and hospitalisation for other cardiac reasons were also gathered.

\section{Cardiopulmonary exercise test}

A maximal symptom-limited treadmill cardiopulmonary exercise test was performed using the modified Bruce protocol (GE Marquette Series 2000 treadmill, Marquette Medical Systems, Inc., Milwaukee, WI, United States of America). Gas analysis was preceded by calibration of the equipment. Minute ventilation, oxygen uptake, and carbon dioxide production were acquired breath-by-breath, using a SensorMedics Vmax 229 gas analyser
(SensorMedics Corporation, Yorba Linda, CA, United States of America). Patients were encouraged to perform exercise until the respiratory exchange ratio was $\geq 1.10$.

Heart rate was measured by continuous electrocardiogram, blood pressure was obtained manually with a sphygmomanometer, and oxygen saturation was monitored by pulse oximetry. Standard spirometry was not available in all patients.

\section{Cardiopulmonary exercise test parameters}

The $\mathrm{pVO}_{2}$ was defined as the highest 30 -second average achieved during exercise and was normalised for body mass. Percentage predicted $\mathrm{pVO}_{2}\left(\mathrm{pVO}_{2}\right.$ predicted (\%)) was calculated from the Wasserman equations. ${ }^{16}$ The $\mathrm{VE} / \mathrm{VCO}_{2}$ slope was calculated by least squares linear regression, using data acquired throughout the whole exercise. ${ }^{17}$

The cardiorespiratory optimal point of ventilation was measured as the minimum value of the ventilatory equivalent for oxygen ( $\mathrm{VE} / \mathrm{VO}_{2}$ minimum), corresponding to the moment during the incremental exercise in which there is less ventilation for a litre of oxygen to be consumed. ${ }^{18}$

The anaerobic threshold was determined by combining the standard methods (V-slope preferentially and ventilatory equivalents). ${ }^{19}$

Partial pressure of end-tidal carbon dioxide $\left(\mathrm{P}_{\mathrm{ET}} \mathrm{CO}_{2}\right)$ was reported before exercise, at anaerobic threshold and at peak exercise in $\mathrm{mmHg}$ units. ${ }^{20}$ Since the increase during exercise until the anaerobic threshold is achieved $\left(\mathrm{P}_{\mathrm{ET}} \mathrm{CO}_{2}\right.$ at anaerobic threshold $-\mathrm{P}_{\mathrm{ET}} \mathrm{CO}_{2}$ at rest) has prognostic importance in heart failure patients, this parameter was also calculated. ${ }^{16}$

Heart rate reserve was calculated as the difference between the maximal heart rate achieved with exercise and the resting heart rate. ${ }^{21}$ Heart rate recovery in the first minute after exercise was defined as the difference between the maximal heart rate achieved with exercise and the heart rate 1 minute into recovery. ${ }^{16}$

Chronotropic incompetence patients were defined as those with a peak heart rate at exercise lower than $80 \%$ of the estimated maximal heart rate $(220$ - age), or lower than $62 \%$ of the estimated maximal heart rate if the patients were prescribed with negative chronotropic agents. ${ }^{22}$

\section{Statistical analysis}

Baseline characteristics were summarised as frequencies (percentages) for categorical variables, as means and standard deviations for continuous variables when normality was verified, and as median and interquartile range when normality was not verified by the Kolmogorov-Smirnov test. The Student's t-test for independent samples or the Mann-Whitney test when normality was not verified was used for the analysis of the variables.

Univariable Cox proportional hazards models were applied, with p-values for time-to-event analyses being based on log-rank tests, and hazard ratios for treatment effects and 95\% confidence intervals presented to study the combined endpoint considering the follow-up time for the different cardiopulmonary exercise test parameters.

The predictive power of several cardiopulmonary exercise test parameters was analysed for the highest area under the curve in the follow-up. ${ }^{23}$ Cut-off values for variables were determined from the receiver operating characteristics curves so that the sum of sensitivity and specificity was maximised. ${ }^{24}$ Hanley and McNeil test was used to compare two correlated receiver operating characteristics curves. $^{25}$ 
Table 1. Baseline characteristics.

\begin{tabular}{|c|c|}
\hline & $\mathrm{n}=44$ \\
\hline Age (years) & $35.1 \pm 8.5$ \\
\hline Male (\%) & $32(72.7 \%)$ \\
\hline Body mass index $\left(\mathrm{kg} / \mathrm{m}^{2}\right)$ & $24.8 \pm 3.8$ \\
\hline NYHA $\geq \mathrm{III}$ & $10(22.8 \%)$ \\
\hline TGA after Senning/Mustard procedure (\%) & $36(81.8 \%)$ \\
\hline CC-TGA (\%) & $8(18.2 \%)$ \\
\hline Pulmonary hypertension (\%) & $12(27.3 \%)$ \\
\hline Cyanotic patients (\%) & $8(18.2 \%)$ \\
\hline Sinus rhythm (\%) & $40(90.9 \%)$ \\
\hline Negative chronotropic agents & $9(20.5 \%)$ \\
\hline Pacemaker & $7(15.9 \%)$ \\
\hline Tricuspid annular plane systolic excursion (mm) & $14.6 \pm 2.8$ \\
\hline At least moderate atrioventricular systemic regurgitation & $9(20.5 \%)$ \\
\hline Hemoglobin $(\mathrm{g} / \mathrm{dL})$ & $15.7 \pm 2.2$ \\
\hline Creatinine $(\mathrm{mg} / \mathrm{dL})$ & $0.8 \pm 0.2$ \\
\hline Sodium (mEq/L) & $139.0 \pm 2.8$ \\
\hline Median BNP $(\mathrm{pg} / \mathrm{ml})$ and interquartile range & $68(41-421)$ \\
\hline \multicolumn{2}{|l|}{ Cardiopulmonary exercise test data } \\
\hline $\mathrm{pVO}_{2}(\mathrm{ml} / \mathrm{kg} / \mathrm{min})$ & $22.9 \pm 6.5$ \\
\hline $\mathrm{pVO}_{2}$ predicted (\%) & $55.8 \pm 13.4$ \\
\hline $\mathrm{VE} / \mathrm{VCO}_{2}$ slope & $35.7 \pm 9.3$ \\
\hline Cardiorespiratory optimal point & $26.5 \pm 5.5$ \\
\hline Duration at anaerobic threshold (seconds) & $499.8 \pm 234.2$ \\
\hline $\mathrm{VO}_{2}$ at anaerobic threshold $(\mathrm{ml} / \mathrm{kg} / \mathrm{min})$ & $16.4 \pm 5.8$ \\
\hline Heart rate at anaerobic threshold (bpm) & $114.5 \pm 25.5$ \\
\hline $\mathrm{P}_{\mathrm{ET}} \mathrm{CO}_{2}$ at anaerobic threshold - initial $\mathrm{P}_{\mathrm{ET}} \mathrm{CO}_{2}$ & $3.7 \pm 5.2$ \\
\hline Heart rate reserve (bpm) & $71.7 \pm 31.4$ \\
\hline Heart rate recovery in the first minute after exercise (bpm) & $21.9 \pm 15.9$ \\
\hline Chronotropic incompetence (\%) & $22(50.0 \%)$ \\
\hline Respiratory exchange ratio & $1.1 \pm 0.1$ \\
\hline $\mathrm{O}_{2}$ saturation at rest $(\%)$ & $93.9 \pm 3.2$ \\
\hline $\mathrm{O}_{2}$ saturation at peak exercise $(\%)$ & $85.0 \pm 10.5$ \\
\hline
\end{tabular}

$\mathrm{cc}-\mathrm{TGA}=$ corrected transposition of the great arteries; TGA $=$ transposition of the great arteries; $\mathrm{BNP}=$ brain natriuretic peptide; $\mathrm{VE}=$ ventilation

Values are mean \pm standard deviation (normal distribution in all characteristics).

Statistical differences with a p-value $<0.05$ were considered significant.

\section{Results}

\section{Overview of TGA patients}

A total of 44 patients were enrolled in the study, 36 (81.8\%) TGA patients previously submitted to a Senning/Mustard procedure and $8(18.2 \%)$ cc-TGA patients. The baseline characteristics are presented in Table 1.

The mean age was $35.1 \pm 8.5$ years, with a higher percentage of males $(72.7 \%)$ in the population. Regarding clinical data, the vast majority of the population were in NYHA class I or II (29.5 and $47.7 \%$, respectively), with eight (18.2\%) cyanotic patients. Medication with negative chronotropic agents was presented in nine $(20.5 \%)$ patients, all of them using beta-blockers. There were only four patients treated with angiotensin-converting enzyme inhibitor or angiotensin receptor blockers (9\%). There were 11 patients $(20 \%)$ on diuretic treatment.

A previous pacemaker implantation was performed with four VVIR and three DDDR pacemaker's systems in seven (15.9\%) patients. The four VVIR system carriers were the only patients in atrial fibrillation during cardiopulmonary exercise test.

Other cardiac anomalies associated with TGA not fully corrected at the time of the cardiopulmonary exercise test included ventricular septal defects (29.5\%), pulmonary stenosis (20.5\%), atrial septal defects $(6.8 \%)$, and atrioventricular septal defect (2.3\%). Regarding previous surgeries, 25 (69.4\%) and 11 (30.6\%) of the TGA patients had a Senning and Mustard procedure, respectively. In cc-TGA patients, only one had one previous surgery for an atrioventricular septal defect correction.

Laboratorial data showed no patient with a glomerular filtration rate lower than $30 \mathrm{ml} / \mathrm{min}$ as well as no patient with anaemia. Brain natriuretic peptide values were highly variable between patients (a median value of $68(41-421) \mathrm{pg} / \mathrm{ml}$ ).

With respect to echocardiographic data, tricuspid annular plane systolic excursion was lower than $16 \mathrm{~mm}$ in $26(68.4 \%)$ patients, with $9(20.5 \%)$ patients with at least moderate A-V systemic regurgitation and no patient with sub-pulmonary ventricle dysfunction.

\section{Primary outcome}

All patients were followed up for at least 12 months from the date of completion of the cardiopulmonary exercise test, with a mean \pm standard deviation follow-up time of $36.7 \pm 26.8$ months. The primary endpoint, a composite of cardiac death or heart failure hospitalisation, occurred in $10(22.7 \%)$ patients (one death and nine heart failure hospitalisations). No patient was lost to follow-up.

\section{Relationship between cardiopulmonary exercise test parameters and primary outcome}

A maximal symptom-limited treadmill cardiopulmonary exercise test was performed using the modified Bruce protocol in all patients, revealing a $\mathrm{pVO}_{2}$ predicted (\%) lower than $50 \%$ in $16(36.3 \%)$ patients. Anaerobic threshold was achieved by $42(95.5 \%)$ patients, but the respiratory exchange ratio was lower than 1.05 and 1.10 in 40.9 and $59.1 \%$, respectively, despite patients were encouraged to perform exercise until the respiratory exchange ratio $\geq 1.10$. There were no complications during the cardiopulmonary exercise test exam, and the patients finished exercising because of fatigue (59.1\%) or dyspnea (40.9\%). Chronotropic incompetence was presented in 24 (54.5\%) patients.

The univariate predictors of the primary outcome by cardiopulmonary exercise test parameters are represented in Table 2, showing that $\mathrm{pVO}_{2}, \mathrm{pVO}_{2}$ (\%) predicted, $\mathrm{VE} / \mathrm{VCO}_{2}$ slope, $\mathrm{P}_{\mathrm{ET}} \mathrm{CO}_{2}$ at peak exercise, heart rate at anaerobic threshold, time until anaerobic threshold, $\mathrm{VO}_{2}$ at anaerobic threshold, $\mathrm{HHR}, \mathrm{O}_{2}$ saturation at peak, and chronotropic index were all predictors of the primary outcome $(\mathrm{p}<0.05$ for all).

Only cardiorespiratory optimal point, heart rate recovery in the first minute after exercise, and the difference between $\mathrm{P}_{\mathrm{ET}} \mathrm{CO}_{2}$ at 
Table 2. Univariate Cox predictors of the primary outcome.

\begin{tabular}{|c|c|c|c|c|}
\hline $\begin{array}{l}\text { Cardiopulmonary exercise test } \\
\text { parameter }\end{array}$ & Wald & $\begin{array}{l}\text { Hazard } \\
\text { ratio }\end{array}$ & $p$-value & $\begin{array}{l}\text { 95\% confidence } \\
\text { interval }\end{array}$ \\
\hline $\mathrm{pVO}_{2}$ & 10.143 & 0.787 & 0.001 & $0.678-0.912$ \\
\hline $\mathrm{pVO}_{2}$ predicted $(\%)$ & 5.442 & 0.935 & 0.020 & $0.884-0.989$ \\
\hline $\mathrm{VE} / \mathrm{VCO}_{2}$ slope & 6.606 & 1.086 & 0.010 & $1.020-1.157$ \\
\hline Optimal point of ventilation & 2.837 & 1.090 & 0.092 & $0.986-1.205$ \\
\hline End-tidal $\mathrm{CO}_{2}$ at $\mathrm{AT}$ - at rest & 2.897 & 0.903 & 0.089 & $0.803-1.016$ \\
\hline End-tidal $\mathrm{CO}_{2}$ at peak & 4.603 & 0.893 & 0.032 & $0.805-0.990$ \\
\hline AT time & 8.530 & 0.994 & 0.003 & $0.991-0.998$ \\
\hline HR at AT & 8.645 & 0.947 & 0.003 & $0.913-0.982$ \\
\hline $\mathrm{VO}_{2}$ at $\mathrm{AT}$ & 10.218 & 0.736 & 0.001 & $0.610-0.888$ \\
\hline HR reserve & 8.533 & 0.976 & 0.003 & $0.960-0.992$ \\
\hline $\begin{array}{l}\text { HR recovery in the first minute } \\
\text { after exercise }\end{array}$ & 3.738 & 0.929 & 0.053 & $0.863-1.001$ \\
\hline $\mathrm{O}_{2}$ saturation at peak & 11.131 & 0.958 & 0.001 & $0.934-0.982$ \\
\hline Chronotropic index & 10.965 & 0.958 & 0.001 & $0.935-0.983$ \\
\hline
\end{tabular}

$\mathrm{AT}=$ anaerobic threshold; $\mathrm{HR}=$ heart rate; $\mathrm{VE}=$ ventilation.

Table 3. AUC analysis.

\begin{tabular}{lcc}
\hline Cardiopulmonary exercise test parameter & $\mathrm{AUC}$ & $\begin{array}{c}95 \% \text { confidence } \\
\text { interval }\end{array}$ \\
\hline $\mathrm{pVO}_{2}$ & 0.838 & $0.696-0.981$ \\
\hline $\mathrm{pVO}_{2}$ predicted (\%) & 0.749 & $0.554-0.994$ \\
\hline $\mathrm{VE} / \mathrm{VCO}_{2}$ slope & 0.713 & $0.506-0.921$ \\
\hline Optimal point of ventilation & 0.729 & $0.554-0.903$ \\
\hline End-tidal $\mathrm{CO}_{2}$ at anaerobic threshold - at rest & 0.769 & $0.585-0.953$ \\
\hline End-tidal $\mathrm{CO}_{2}$ at peak & 0.745 & $0.584-0.906$ \\
\hline Anaerobic threshold time & 0.818 & $0.630-1.000$ \\
\hline Heart rate at anaerobic threshold & 0.864 & $0.716-1.000$ \\
\hline VO ${ }_{2}$ at anaerobic threshold & 0.807 & $0.627-0.987$ \\
\hline Heart rate reserve & 0.779 & $0.623-0.936$ \\
\hline Heart rate recovery in the first minute after & 0.675 & $0.460-0.890$ \\
\hline exercise & & \\
\hline $\mathrm{O}_{2}$ saturation at peak & 0.830 & $0.700-0.959$ \\
\hline Chronotropic index & 0.802 & $0.649-0.955$ \\
\hline
\end{tabular}

$\mathrm{AUC}=$ area under the curve; $\mathrm{VE}=$ ventilation.

anaerobic threshold and rest did not achieve significant statistical difference ( $\mathrm{p}$ between 0.05 and 0.10 ).

In addition to the Cox analysis, these cardiopulmonary exercise test parameters were analysed for the highest area under the curve in the follow-up period (Table 3).

Heart rate at anaerobic threshold had the highest area under the curve value (0.864), followed by $\mathrm{pVO}_{2}(0.838)$ and $\mathrm{O}_{2}$ saturation at peak exercise (0.830). With the exception of heart rate recovery in the first ${ }^{t}$ minute after exercise (0.675), all parameters analysed had an area under the curve value higher than 0.700 .

Since four $(9.1 \%)$ patients were in atrial fibrillation, a subanalysis was performed only with patients in sinus rhythm without
Table 4. Sensitivity and specificity results.

\begin{tabular}{|c|c|c|c|}
\hline $\begin{array}{l}\text { Cardiopulmonary exercise test } \\
\text { parameter }\end{array}$ & $\begin{array}{l}\text { Best } \\
\text { value }\end{array}$ & $\begin{array}{c}\text { Sensitivity } \\
(\%)\end{array}$ & $\begin{array}{l}\text { Specificity } \\
(\%)\end{array}$ \\
\hline $\mathrm{pVO}_{2}(\mathrm{ml} / \mathrm{kg} / \mathrm{min})$ & $<20.0$ & 80.0 & 76.5 \\
\hline $\mathrm{pVO}_{2}$ predicted (\%) & $<50 \%$ & 70.0 & 67.6 \\
\hline $\mathrm{VE} / \mathrm{VCO}_{2}$ slope & $>37.0$ & 87.5 & 67.6 \\
\hline Optimal point of ventilation & $>28.0$ & 77.8 & 73.5 \\
\hline End-tidal $\mathrm{CO}_{2}$ at $A T^{2}$ - at rest $(\mathrm{mmHg})$ & $<1.8$ & 71.4 & 73.5 \\
\hline$A T^{2}$ time (min) & $<7$ & 87.5 & 83.5 \\
\hline Heart rate at $\mathrm{AT}^{2}$ (bpm) & $<95$ & 87.5 & 82.4 \\
\hline $\mathrm{VO}_{2}$ at $A T^{2}(\mathrm{ml} / \mathrm{kg} / \mathrm{min})$ & $<15.0$ & 87.5 & 83.5 \\
\hline Heart rate reserve (bpm) & $<70$ & 70.0 & 76.5 \\
\hline $\mathrm{O}_{2}$ saturation at peak $(\%)$ & $<86 \%$ & 88.9 & 69.0 \\
\hline Chronotropic index (\%) & $<78 \%$ & 88.9 & 62.5 \\
\hline
\end{tabular}

AT $=$ anaerobic threshold; $\mathrm{VE}=$ ventilation.

an increase in the area under the curve value of heart rate at anaerobic threshold (0.853).

Since $\mathrm{pVO}_{2}$ prognosis power had been related to the need to perform a truly maximum cardiopulmonary exercise test, as assessed by a respiratory exchange ratio $>1.05,{ }^{20}$ a sub-analysis was performed showing an area under the curve value of 0.829 (CI $0.608-1.000$ ) in patients with a respiratory exchange ratio $>1.05$.

Another sub-analysis was performed regarding $\mathrm{VE} / \mathrm{VCO}_{2}$ slope excluding cyanotic patients, since previous studies have shown a lack of correlation between $\mathrm{VE} / \mathrm{VCO}_{2}$ slope and prognosis in this group of patients, ${ }^{26,27}$ without a significant decrease in the AUC value.

Cut-off values for variables were determined from the ROC curves so that the sum of sensitivity and specificity was maximised and are presented in Table 4.

Reaching anaerobic threshold before the 7 minute of exercise and $\mathrm{VO}_{2}$ at anaerobic threshold $\leq 15 \mathrm{ml} / \mathrm{kg} / \mathrm{min}$ had a sensitivity of $87.5 \%$ and a specificity of $83.5 \%$ for the primary outcome. The most used cardiopulmonary exercise test parameters regarding prognosis in heart failure had a sensitivity of 80.0 and $87.5 \%$ and a specificity of 76.5 and $67.6 \%$, respectively, for $\mathrm{pVO}_{2}$ $\leq 20 \mathrm{ml} / \mathrm{kg} / \mathrm{min}$ and $\mathrm{VE} / \mathrm{VCO}_{2}$ slope $>37.0$, slightly lower than the values achieved with anaerobic threshold time, heart rate at anaerobic threshold, and $\mathrm{VO}_{2}$ at anaerobic threshold. No differences were found in the analysis excluding atrial fibrillation patients.

The Hanley and McNeil test was applied for comparing each area under the curve parameters, with no statistically significant difference found.

\section{Discussion}

In a group of adult TGA patients with systemic circulation supported by the morphological right ventricle and evidence of limitation in their exercise capacity, as shown by baseline mean values of $\mathrm{pVO}_{2}$ predicted (\%) of $55.8 \pm 13.4 \%$, several cardiopulmonary exercise test parameters were predictors of the primary outcome of cardiac death or heart failure hospitalisation, including $\mathrm{pVO}_{2}$, $\mathrm{pVO}_{2}(\%)$ predicted, $\mathrm{VE} / \mathrm{VCO}_{2}$ slope, $\mathrm{P}_{\mathrm{ET}} \mathrm{CO}_{2}$ at peak exercise, heart rate at anaerobic threshold, time until anaerobic threshold, $\mathrm{VO}_{2}$ at anaerobic threshold, heart rate reserve, $\mathrm{O}_{2}$ saturation at 
peak, and chronotropic index (all with a $\mathrm{p}<0.05$ ), while cardiorespiratory optimal point, heart rate recovery in the first minute after exercise, and the difference between $\mathrm{P}_{\mathrm{ET}} \mathrm{CO}_{2}$ at anaerobic threshold and rest did not achieve statistical significance ( $\mathrm{p}$ between 0.05 and 0.10 ).

In heart failure patients, cardiopulmonary exercise test is a powerful predictor of mortality and is used as the standard criterion for the need for heart transplantation, ${ }^{11}$ with $\mathrm{pVO}_{2}$ and $\mathrm{VE} / \mathrm{VCO}_{2}$ slope as the most used risk assessment tools. ${ }^{12}$ Several trials had tried a similar approach to predict survival in CHD patients, showing that $\mathrm{pVO}_{2}$ and $\mathrm{VE} / \mathrm{VCO}_{2}$ slope are related to midterm survival in this patients. 13,14

In a previous investigation, $\mathrm{VE} / \mathrm{VCO}_{2}$ slope was the most powerful exercise predictor of mortality on non-cyanotic adult CHD patients. ${ }^{26}$ However, some studies had not shown the same prognostic prediction with $\mathrm{VE} / \mathrm{VCO}_{2}$ slope in cyanotic patients, ${ }^{14,26,27}$ suggesting that cyanosis is associated with raised $\mathrm{VE} / \mathrm{VCO}_{2}$ slope, but these higher values should not be extrapolated alone to predict a worse prognosis. In our study, despite being a univariate predictor of the primary outcome $(\mathrm{p}=0.010), \mathrm{VE} /$ $\mathrm{VCO}_{2}$ slope only achieved an area under the curve value of 0.713 , numerically lower than other analysed parameters like $\mathrm{pVO}_{2}(0.838)$ and heart rate at anaerobic threshold (0.864). Since $18.2 \%$ of the patients were cyanotic, a sub-analysis was performed in non-cyanotic patients, without an increase in the area under the curve value (0.703).

Of all the parameters analysed, $\mathrm{pVO}_{2}$ had the second highest area under the curve value $(0.838)$, with a value lower than $20 \mathrm{ml} / \mathrm{kg} / \mathrm{min}$ achieving a sensitivity of $80.0 \%$ and a specificity of $76.5 \%$ for the primary outcome. As well as other cardiopulmonary exercise test parameters, $\mathrm{pVO}_{2}$ had been related to the need to perform a truly maximum cardiopulmonary exercise test, as assessed by a respiratory exchange ratio $>1.05 .{ }^{20}$ However, in our population, respiratory exchange ratio was lower than 1.05 in $40.9 \%$, so we performed a sub-analysis for $\mathrm{pVO}_{2}$ in patients with respiratory exchange ratio $>1.05$ which showed an area under the curve value of 0.829 , numerically lower than the 0.838 achieved with all patients.

Anaerobic threshold represents the moment where anaerobic metabolism becomes relevant for the production of the amount of energy demanded by exercise. ${ }^{19}$ Previous studies in CHD patients have shown that $\mathrm{VO}_{2}$ at anaerobic threshold values is below normal $\left(45-65 \%\right.$ of the $\left.\mathrm{pVO}_{2}\right){ }^{28}$ and some may fail to achieve anaerobic threshold in a way similar to heart failure patients. ${ }^{29}$ In our trial, $\mathrm{VO}_{2}$ at anaerobic threshold values was $55.8 \pm 13.4 \%$ of the $\mathrm{pVO}_{2}$, and only two $(4.5 \%)$ patients failed to achieve anaerobic threshold, a lower percentage than previous trials with all types of CHD.

Parameters related to anaerobic threshold (anaerobic threshold time $<7$ minute of exercise, heart rate at anaerobic threshold $<95 \mathrm{bpm}$, and $\mathrm{VO}_{2}$ at anaerobic threshold $<15.0 \mathrm{ml} / \mathrm{kg} / \mathrm{min}$ ) reached the highest values of sensitivity $(87.5 \%)$ and specificity $(82.4-83.5 \%)$ for the prediction of the primary outcome, suggesting that they should be used in the routine evaluation of these patients to stratify the risk of cardiac death or heart failure hospitalisation.

Chronotropic incompetence was previously related to mortality in adult CHD patients ${ }^{21}$ and is common in this group patients $\left(30-60 \%\right.$ of the patient $\left.\mathrm{s}^{27}\right)$, since they usually have a blunted heart response to exercise related to autonomic dysfunction, cardiac arrhythmias, and high use of chronotropic negative medication, like beta-blockers. ${ }^{21,30}$ However, some trials have shown that beta-blockers have beneficial effects in systemic right ventricular dysfunction, ${ }^{31,32}$ being the medication with the most supportive data for TGA patients with systemic right ventricle dysfunction. Medication with beta-blockers was presented in nine (20.5\%) patients, which may be explained by a mean tricuspid annular plane systolic excursion of $14.6 \pm 2.8 \mathrm{~mm}$, and there was no relationship regarding the use of beta-blockers to the primary outcome. Additionally, a previous pacemaker implantation was performed in seven (15.9\%) patients; however, all of them had a rate responsive function.

In our study, chronotropic incompetence was present in 24 (54.4\%) patients, with a mean chronotropic index of $72.0 \pm 20.6 \%$. Chronotropic index had an area under the curve value of 0.802 , with a value lower than $78 \%$ with high sensitivity (88.9\%) but lower specificity (62.5\%) for the primary outcome.

Other heart rate-related parameters (heart rate reserve; heart rate recovery in the first minute after exercise) had shown prognostic importance in CHD patients. ${ }^{14,15}$ In our trial, heart rate reserve as opposed to heart rate recovery in the first minute after exercise was a significant predictor of the primary outcome $(p=0.003$ versus $p=0.053$ ). Other parameters had higher values of sensitivity and specificity than heart rate reserve and heart rate recovery in the first minute after exercise, which could be explained by the confound effect of patients doing beta-blockers and with previous pacemaker implantation. Few patients $(9.1 \%)$ were in atrial fibrillation.

Despite a previous trial showing that cardiorespiratory optimal point is a good predictor of all-cause mortality in adults, ${ }^{33}$ in our trial cardiorespiratory optimal point did not achieve statistical significance for the prediction of the outcome $(\mathrm{p}=0.092)$. Likewise, the increase in $\mathrm{P}_{\mathrm{ET}} \mathrm{CO}_{2}$ until the anaerobic threshold is achieved $\left(\mathrm{P}_{\mathrm{ET}} \mathrm{CO}_{2}\right.$ at anaerobic threshold $-\mathrm{P}_{\mathrm{ET}} \mathrm{CO}_{2}$ at rest), which was previously shown to have prognostic importance in heart failure patients ${ }^{16}$ did not achieve statistical significance neither $(p=0.089)$. To the best of our knowledge, this was the first time that cardiorespiratory optimal point was assessed in a CHD population.

Uncertainty remains about managing systemic right ventricular failure $^{8}$ and heart transplantation currently remains the only longterm life-saving procedure. ${ }^{4}$ Despite a number of confounders that are less relevant in heart failure, cardiopulmonary exercise test was able to predict heart failure events in this TGA population with systemic right ventricle. These confounders include a high number of patients with a respiratory exchange ratio lower than 1.05 (40.9\%), which may favour the use of sub-maximal exercise parameters such as $\mathrm{VO}_{2}$ at anaerobic threshold rather than maximal effort-dependent parameters such as $\mathrm{pVO}_{2}$. However, when the Hanley and McNeil test was applied for comparing each area under the curve parameters, no statistically significant difference was found, which is not surprising since previous studies have already shown that the usual benchmarks for interpretation of respiratory exchange ratio may not have the same meaning in patients with $\mathrm{CHD} .^{34}$

\section{Study limitations}

There are limitations to our study that should be referred when interpreting our results. This was a single-centre retrospective experience with only 44 patients, and therefore, the results can reflect local practice and might not represent other adult CHD centres. Despite being a small study, to the best of our knowledge, it is the first study addressing the use of cardiopulmonary exercise test to stratify the risk of heart failure events in TGA patients with systemic right ventricle alone without mixing other $\mathrm{CHD}$ and 
showed promising results for the use of cardiopulmonary exercise test to predict heart failure events in this population.

Mustard/Senning physiology and response to exercise may not be completely similar to cc-TGA patients despite having a systemic right ventricle, since atrial baffles cause a relative preload reduction during exercise when compared to cc-TGA patients. ${ }^{35}$ However, the results would be similar if we only analysed the 36 atrial switch operation patients.

The primary outcome was a composite of cardiac death and heart failure hospitalisation. Although heart failure hospitalisation is not as important as cardiac death for the prognosis of the patients, heart failure hospitalisation is a major hazard for adult CHD patients and is associated with substantial costs and an amplified risk of subsequent mortality, ${ }^{13,36,37}$ so we used it to increase the percentage of the primary outcome since cardiac mortality was low in the mean 3-year follow-up (2.3\%).

Each cardiopulmonary exercise test parameter was analysed for the area under the curve and the cut-off value so that the sum of sensitivity and specificity was maximised. However, some interaction between cardiopulmonary exercise test parameters cannot be excluded, and the results cannot be interpreted as if each cardiopulmonary exercise test parameter has a prognosis power that can be added to the predictive power of other cardiopulmonary exercise test parameters.

Several cardiopulmonary exercise test parameters had been related to the need to perform a truly maximum cardiopulmonary exercise test, as assessed by a respiratory exchange ratio $>1.05{ }^{20}$ However, in our population, the respiratory exchange ratio was lower to 1.05 in $40.9 \%$, so we decided to perform a sub-analysis for $\mathrm{pVO}_{2}$ in patients with a respiratory exchange ratio $>1.05$ which showed an area under the curve value of 0.829 , no higher than the 0.838 achieved with all patients. Additionally, we used several parameters ( $\mathrm{VE} / \mathrm{VCO}_{2}$ slope, cardiorespiratory optimal point, and $\mathrm{P}_{\mathrm{ET}} \mathrm{CO}_{2}$ at anaerobic threshold $-\mathrm{P}_{\mathrm{ET}} \mathrm{CO}_{2}$ at rest) that do not need a maximal exercise cardiopulmonary exercise test to have prognostic power. ${ }^{16}$

In a similar way, another sub-analysis was performed regarding $\mathrm{VE} / \mathrm{VCO}_{2}$ slope excluding cyanotic patients, since previous studies have shown a lack of correlation between $\mathrm{VE} / \mathrm{VCO}_{2}$ slope and prognosis in this group of patients, ${ }^{26,27}$ with an area under the curve value of 0.703 , no higher than the 0.713 achieved with all patients.

\section{Conclusions}

Patients with both TGA previously submitted to a Senning/ Mustard procedure and cc-TGA have right ventricle working against systemic pressures, thereby rendering these patients to an increased risk for heart failure events. Heart rate at anaerobic threshold had the highest predictive power of all cardiopulmonary exercise test parameters analysed, with a value $\leq 95 \mathrm{bpm}$ achieving a sensitivity of $87.5 \%$ and a specificity of $82.4 \%$ for the primary outcome. Additionally, in a way similar to heart failure patients, several cardiopulmonary exercise test parameters were able to provide prognostic information regarding heart failure events, suggesting that cardiopulmonary exercise test should be used in the routine evaluation of these patients as a prognostic tool.

Acknowledgements. We sincerely thank Centro Hospitalar Universitário Lisboa Central for the opportunity to perform this study.

Financial Support. This research received no specific grant from any funding agency, commercial, or not-for-profit sectors.
Conflicts of Interest. None

\section{References}

1. Villafane J, Lantin-Hermoso MR, Bhatt AB, et al. D-transposition of the great arteries: the current era of the arterial switch operation. J Am Coll Cardiol 2014; 64: 498-511.

2. Wallis GA, Debich-Spicer D, Anderson RH. Congenitally corrected transposition. Orphanet J Rare Dis 2011; 6: 22.

3. Shah S, Gupta T, Ahmad R. Managing heart failure in transposition of the great arteries. Ochsner J 2015; 15: 290-296.

4. Filippov AA, Del Nido PJ, Vasilyev NV. Management of systemic right ventricular failure in patients with congenitally corrected transposition of the great arteries. Circulation 2016; 134: 1293-1302.

5. Lopez L, Cohen MS, Anderson RH, et al. Unnatural history of the right ventricle in patients with congenitally malformed hearts. Cardiol Young 2010; 20 (Suppl 3): 107-112.

6. Weber KT, Brilla CG. Pathological hypertrophy and cardiac interstitium. Fibrosis and renin-angiotensin-aldosterone system. Circulation 1991; 83: 1849-1865.

7. Hauser M, Bengel FM, Hager A, et al. Impaired myocardial blood flow and coronary flow reserve of the anatomical right systemic ventricle in patients with congenitally corrected transposition of the great arteries. Heart 2003; 89: 1231-1235.

8. Winter MM, Bouma BJ, Groenink M, et al. Latest insights in therapeutic options for systemic right ventricular failure: a comparison with left ventricular failure. Heart 2009; 95: 960-963.

9. Hauser M, Meierhofer C, Schwaiger M, Vogt M, Kaemmerer H, Kuehn A. Myocardial blood flow in patients with transposition of the great arteries risk factor for dysfunction of the morphologic systemic right ventricle late after atrial repair. Circ J 2015; 79: 425-431.

10. Said SM, Burkhart HM, Schaff HV, Dearani JA. Congenitally corrected transposition of great arteries: surgical options for the failing right ventricle and/or severe tricuspid regurgitation. World J Pediatr Congenital Heart Surg 2011; 2: 64-79.

11. Mehra MR, Canter CE, Hannan MM, et al. The 2016 International Society for Heart Lung Transplantation listing criteria for heart transplantation: a 10-year update. J Heart Lung Transplant 2016; 35: 1-23.

12. Butler J, Khadim G, Paul KM, et al. Selection of patients for heart transplantation in the current era of heart failure therapy. J Am Coll Cardiol 2004; 43: 787-793.

13. Diller GP, Dimopoulos K, Okonko D, et al. Exercise intolerance in adult congenital heart disease: comparative severity, correlates, and prognostic implication. Circulation 2005; 112: 828-835.

14. Inuzuka R, Diller GP, Borgia F, et al. Comprehensive use of cardiopulmonary exercise testing identifies adults with congenital heart disease at increased mortality risk in the medium term. Circulation 2012; 125: 250-259.

15. Mantegazza V, Apostolo A, Hager A. Cardiopulmonary exercise testing in adult congenital heart disease. Ann Am Thorac Soc 2017; 14 (Suppl 1): S93-S101.

16. Guazzi M, Arena R, Halle M, Piepoli MF, Myers J, Lavie CJ. 2016 Focused update: clinical recommendations for cardiopulmonary exercise testing data assessment in specific patient populations. Circulation 2016; 133: e694-e711.

17. Clark AL, Poole-Wilson PA, Coats AJ. Relation between ventilation and carbon dioxide production in patients with chronic heart failure. J Am Coll Cardiol 1992; 20: 1326-1332.

18. Ramos PS, Ricardo DR, Araujo CG. Cardiorespiratory optimal point: a submaximal variable of the cardiopulmonary exercise testing. Arq Bras Cardiol 2012; 99: 988-996.

19. Wasserman K, Stringer WW, Casaburi R, Koike A, Cooper CB. Determination of the anaerobic threshold by gas exchange: biochemical considerations, methodology and physiological effects. Z Kardiol 1994; 83 (Suppl 3): 1-12.

20. Balady GJ, Arena R, Sietsema K, et al. Clinician's guide to cardiopulmonary exercise testing in adults: a scientific statement from the American Heart Association. Circulation 2010; 122: 191-225. 
21. Diller GP, Dimopoulos K, Okonko D, et al. Heart rate response during exercise predicts survival in adults with congenital heart disease. J Am Coll Cardiol 2006; 48: 1250-1256.

22. Khan MN, Pothier CE, Lauer MS. Chronotropic incompetence as a predictor of death among patients with normal electrograms taking beta blockers (metoprolol or atenolol). Am J Cardiol 2005; 96: 1328-1333.

23. Heagerty PJ, Lumley T, Pepe MS. Time-dependent ROC curves for censored survival data and a diagnostic marker. Biometrics 2000; 56: 337-344.

24. Bewick V, Cheek L, Ball J. Statistics review 13: receiver operating characteristic curves. Crit Care 2004; 8: 508-512.

25. Hanley JA, McNeil BJ. The meaning and use of the area under a receiver operating characteristic (ROC) curve. Radiology 1982; 143: 29-36.

26. Dimopoulos K, Okonko DO, Diller GP, et al. Abnormal ventilatory response to exercise in adults with congenital heart disease relates to cyanosis and predicts survival. Circulation 2006; 113: 2796-2802.

27. Khan AM, Paridon SM, Kim YY. Cardiopulmonary exercise testing in adults with congenital heart disease. Expert Rev Cardiovasc Ther 2014; 12: 863-872.

28. Reybrouck T, Rogers R, Weymans M, et al. Serial cardiorespiratory exercise testing in patients with congenital heart disease. Eur J Pediatr 1995; 154: 801-806.

29. Kadish A, Nademanee K, Volosin K, et al. A randomized controlled trial evaluating the safety and efficacy of cardiac contractility modulation in advanced heart failure. Am Heart J 2011; 161: 329-337 e1-2.
30. Reybrouck T, Vangesselen S, Gewillig M. Impaired chronotropic response to exercise in children with repaired cyanotic congenital heart disease. Acta Cardiol 2009; 64: 723-727.

31. Bouallal R, Godart F, Francart C, Richard A, Foucher-Hossein C, Lions C. Interest of beta-blockers in patients with right ventricular systemic dysfunction. Cardiol Young 2010; 20: 615-619.

32. Doughan AR, McConnell ME, Book WM. Effect of beta blockers (carvedilol or metoprolol XL) in patients with transposition of great arteries and dysfunction of the systemic right ventricle. Am J Cardiol 2007; 99: 704-706.

33. Ramos PS, Araujo CG. Cardiorespiratory optimal point during exercise testing as a predictor of all-cause mortality. Rev Port Cardiol 2017; 36: 261-269.

34. Glaser S, Opitz CF, Bauer U, et al. Assessment of symptoms and exercise capacity in cyanotic patients with congenital heart disease. Chest 2004; 125 : 368-376.

35. Fratz S, Hager A, Busch R, et al. Patients after atrial switch operation for transposition of the great arteries can not increase stroke volume under dobutamine stress as opposed to patients with congenitally corrected transposition. Circ J 2008; 72: 1130-1135.

36. Moons P, Siebens K, De Geest S, Abraham I, Budts W, Gewillig M. A pilot study of expenditures on, and utilization of resources in, health care in adults with congenital heart disease. Cardiol Young 2001; 11: 301-313.

37. Kaemmerer H, Fratz S, Bauer U, et al. Emergency hospital admissions and three-year survival of adults with and without cardiovascular surgery for congenital cardiac disease. J Thorac Cardiovasc Surg 2003; 126: 1048-1052. 